\title{
Cassini/Huygens Mission To Saturn: Results And Prospects
}

\author{
Dennis L. Matson ${ }^{1}$, Jean-Pierre Lebreton ${ }^{2}$, and Linda Spilker ${ }^{1}$ \\ 1 Jet Propulsion Laboratory, California Institute of Technology, 4800 \\ Oak Grove Drive, Pasadena, CA 91109., USA \\ ${ }^{2}$ Research and Scientific Support Department, ESA/ESTEC, P.O. Box \\ 299,2200 AG Noordwijk, The Netherlands, dmatson@jpl.nasa.gov
}

The Cassini spacecraft was launched in October, 1997. Since then it has been on an interplanetary trajectory aimed toward Saturn and arriving there on July 1, 2004. En route, Cassini has flown by Venus, the Earth, and Jupiter. Each of these events yielded new scientific results. (e.g., 11 papers in J. Geophys. Res. 106, 30099-30279.) The Cassini flyby of Jupiter, with Galileo already in Jovian orbit, enabled the first-ever simultaneous measurements by two spacecraft at an outer planet. This fortuitous event provided a unique opportunity to investigate the giant planet's magnetic field and the properties of the Jovian system. It provided a focused period for intensive observations of Jupiter and cooperation with investigators using Galileo, Hubble, Chandra, and ground-based observatories. The results achieved at Jupiter were stunning (e.g., 8 articles in Nature 415, 965-1005, February 28, 2002). Recent results and the current status of the spacecraft and mission will be discussed. Of note are the dates of July 1, 2004 when Cassini goes into orbit about Saturn and January 14, 2005 when Huygens enters the atmosphere of Titan. The Cassini/Huygens mission is a joint undertaking by NASA and ESA, with ASI as a partner via a bilateral agreement with NASA. 